\section{Der Herausforderung begegnen}

\section{Zukunftsszenarien für die Intensivmedizin}

Die Menschen werden immer älter, manche von ihnen - nicht zuletzt durch die Entwicklungen der modernen Medizin leben immer länger auch mit schwerer Erkrankung. Für die Intensivmedizin stellt dies besondere Herausforderungen dar. Das Österreichische Zentrum für Dokumentation und Qualitätssicherung in der Intensivmedizin (ASDI) beschäftigt sich seit beinahe 20 Jahren damit, die bestmögliche Behandlung der Schwerkranken sicherzustellen und präsentierte beim jährlichen Meeting Ende November in Wien aktuelle Themen und mögliche Lösungen.

Gleichzeitig mit dem medizinischen Fortschritt, so das ASDI, hat das Krankenanstalten-Arbeitszeitgesetz, das schrittweise die Arbeitszeit der Ärzte limitieren soll, die Präsenz der Ärzte im Spital reduziert. Wobei das Arbeitszeitgesetz freilich für alle Gesundheitsberufe gilt. „Der Druck wächst“, stellte Univ.-Prof. DDr. Philipp Metnitz, Leiter der Klinischen Abteilung für Allgemeine Anästhesiologie, Notfall- und Intensivmedizin am LKH Universitätsklinikum Graz, fest: „Wir vom ASDI betrachten es als unsere Aufgabe, die geänderten Rahmenbedingungen als Chance zu be- greifen und das Beste daraus zu machen.“ Überarbeitete Spitalsmitarbeiter stellen jedenfalls nicht nur für die Patienten, sondern auch für sich selbst eine Gefahr dar.

Als ganz besondere Belastung mit entsprechend negativen Folgen nannte Prim. Univ.-Prof. Dr. Andreas Valentin, Leiter der Abteilung für Innere Medizin am Kardinal Schwarzenberg'schen Krankenhaus in Schwarzach/Pongau, Salzburg, und Präsident der ÖGIAIN (Österreichische Gesellschaft für Internistische und Allgemeine Intensivmedizin und Notfallmedizin) das akute Schlafdefizit: „24 Stunden ohne Schlaf wirken wie ein Promille Alkohol im Blut. Die Leistungsfähigkeit nimmt ab, die Fehlerquoten steigen an." Auch bei chronischer Übermüdung stellen sich ähnliche Wirkungen ein. Studien zeigen, dass bei besonders hoher Arbeitsbelastung der Ärzte die Mortalität an Intensivstationen bis zum Dreifachen ansteigen kann. Bei Überlastung von Pflegepersonen ist z.B. ein signifikanter Anstieg der Infektionsraten zu beobachten. Im Rahmen der Arbeitsüberlastung kommt es auch zu einer falschen Selbsteinschätzung der Betroffenen. Der Aussage „Auch wenn ich übermüdet bin, erbringe ich in kritischen Phasen eine gleichwertige Leis- tung." stimmen 64 Prozent der Intensivmediziner und der Intensivpflegepersonen zu. Piloten sind in ihrer Selbsteinschätzung offenbar realistischer, hier beträgt die Zustimmungsrate nur 26 Prozent.

Kürzere Arbeitszeiten führen zu häufigeren Dienstübergaben - und einem erhöhten Informationsverlust. Um die Kontinuität der Patientenbetreuung zu wahren, sind daher streng strukturierte Übergaben anhand von Checklisten und Protokollen notwendig. „Wir haben uns mit der Kontinuität sehr eingehend beschäftigt, weil wir wissen, dass bei höherer Kontinuität die Patienten kürzer auf der Intensivstation verbleiben und die Mortalität geringer ist", berichtete Univ.-Prof. Dr. J. Michael Hiesmayr, Leiter der Klinischen Abteilung für Herz-Thorax-Gefäßchirurgische Anästhesie und Intensivmedizin am AKH, Medizinische Universität Wien: „Für eine optimale Dienstübergabe müssen beide Seiten aktiv werden - der Übergeber ebenso wie der Empfänger. Bestimmte Abläufe, Inhalte etc. sind genau einzuhalten. Das muss man trainieren!"

Als einen der Punkte, um die Versorgung bei höherem Patientenaufkommen und reduzierter Arbeitszeit gewährleisten zu können, nannte Hiesmayr auch die Stärkung der Pflegeberufe: Definierte Kompetenzen für Pflegepersonen - gezieltere Ausbildungen - verstärkte Motivation junger Menschen, anstelle von weit verbreiteten Berufen den Pflegeberuf zu ergreifen.

Quelle: Presseinformation ASDI

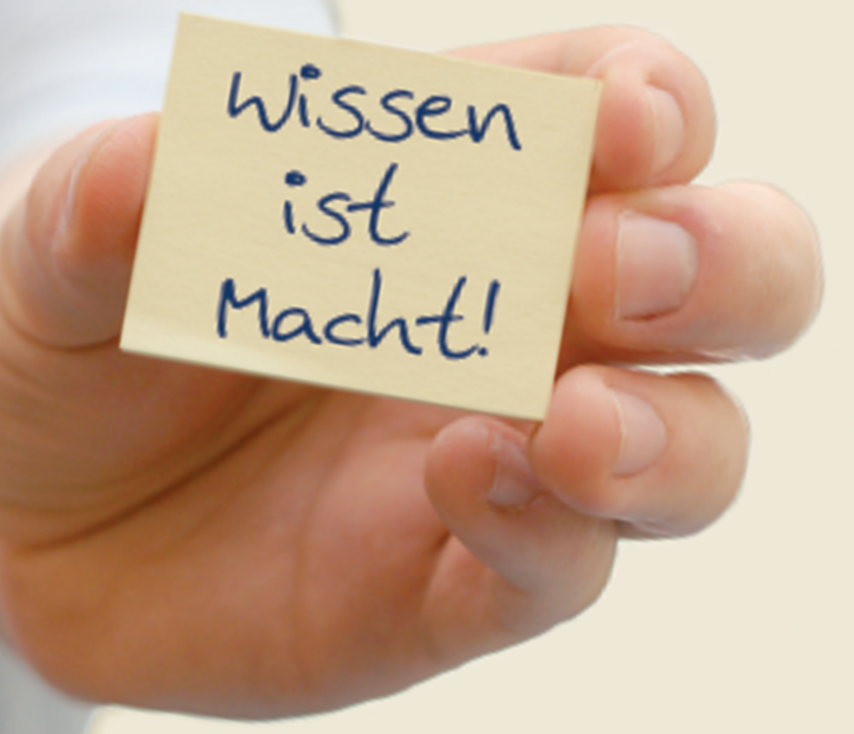

MSc Pflegemanagement

Beginn: 15. März 2016

Dauer: 6 Semester berufsbegleitend, 4 Semester Vollzeit

Gebühr: EUR 9.600,-

Abschluss: Master of Science (MSc)

Kontakt: sophie.schwed@donau-uni.ac.at, Tel. +43 (0)2732 893-2745

Wundmanagement

Beginn: 29. Februar 2016

Dauer: 1 Semester/2 Semester berufsbegleitend

Gebühr: EUR 2.850,-/4.100,-

Abschluss: Zertifikat/Akademische/r Wundmanager/in

Kontakt: christiane.gfoehler@donau-uni.ac.at, Tel. +43 (0)2732 893-2746 